\title{
Surface Segregation Analysis of Hydrophobic Additive of Non-topcoat Resist
}

\author{
Takuya Hagiwara ${ }^{1}$, Takeo Ishibashi ${ }^{1}$, Mamoru Terai ${ }^{2}$, Teruhiko Kumada ${ }^{2}$, \\ Naoko Shirota ${ }^{3}$, Osamu Yokokoji ${ }^{3}$, Takeshi Matsunobe ${ }^{4}$, Naoki Man $^{4}$, \\ Kazuhiro Yoshikawa ${ }^{4}$, Yusaku Tanahashi ${ }^{4}$, and Tetsuro Hanawa ${ }^{1}$ \\ ${ }^{1}$ Renesas Technology Corp., 4-1, Mizuhara, Itami-shi, Hyogo 664-0005, Japan \\ ${ }^{2}$ Mitsubishi Electric Corp., 8-1-1 Honmachi, Tsukaguchi, Amagasaki-shi, Hyogo 661-8661, Japan \\ ${ }^{3}$ Asahi Glass Co., Ltd., 1150, Hazawa-cho, Kanagawa-ku, Yokohama-shi, \\ Kanagawa 221-8755, Japan \\ ${ }^{4}$ Toray Research Center Inc., 3-3-7, Otsu, Shiga 525-8567, Japan
}

\begin{abstract}
Non-topcoat (non-TC) resists, which blend hydrophobic additives into a resist polymer, have been proposed by vendors. To minimize the surface free energy, a hydrophobic additive segregates to the surface and forms a layer. The improvement of surface hydrophobicity and the suppression of resist component leaching are achieved by using this segregation layer. Segregation is an unstable physical phenomenon that is influenced by the environment. Hence, the characteristics of segregation layers must be sufficiently understood to use non-TC resists safely and have been thoroughly investigated using various analyses in this study. The results revealed the following: (1) A segregation layer comprising only the hydrophobic additive could form if the additive had low critical surface tension. However, the segregation layer differed from the coating film and exhibited a film density comparable to that of the base polymer. (2) The segregation mostly occurred during spin coating. (3) A non-TC resist should have the optimum film thickness: a thin film exhibited low hydrophobicity, whereas the additive remained in the resist bulk for a thick film. (4) There were no differences in the segregation layer in the central and outer parts of the wafer. When using a non-TC resist, it is necessary to understand the characteristics and note the usage. If it is used with sufficient understanding, it is sure that a non-TC resist can reduce the cost and increase the throughput safely.
\end{abstract}

Keyword: non-topcoat resist, hydrophobic additive, segregation phenomenon

\section{Introduction}

193-nm immersion (193i) lithography is being applied to the $45-\mathrm{nm}$ node of mass products $[1,2]$. In the $193 \mathrm{i}$ lithography, the space between the projection lens and the wafer in a tool is filled with deionized water. A local fill system is adopted for the present immersion lithography so that the water is not polluted by coming in contact with many areas. In order to keep the water in the local fill and to enable scanning exposure, the substrate surface requires high hydrophobicity. Moreover, it is necessary to suppress leaching of resist components into the water so that the projection lens may not be polluted through the water. In order to meet these requirements, a topcoat film with a fluorine compound is coated on the resist [3-5]. An alkaline-soluble topcoat is widely used for the convenience that it can be removed in a normal development process.

However, a topcoat is expensive. In addition, an alkali-soluble topcoat has less hydrophobicity because it contains a polar unit in the polymer.

Non-topcoat (non-TC) resists have been proposed by vendors [6,7]. A non-TC resist is a resist that contains a hydrophobic additive. 
To minimize surface free energy, the hydrophobic additive segregates to the surface and forms a layer. The improvement of surface hydrophobicity and the suppression of resist component leaching aim to be achieved by using this segregation layer. Because the topcoat becomes unnecessary and the amount of a hydrophobic additive required is much less than that of the polymer of a coating film such as a topcoat, it is possible to assure cost reduction. For defect reduction, this additive should be soluble in an alkaline developer. However, because the additive amount is small, the solubility need not be as high as that an alkali-soluble topcoat is required to have. Therefore, a non-TC resist can have hydrophobicity higher than that of an alkaline-soluble topcoat. Although a topcoat is more advantageous for the suppression of resist component leaching, it is considered that there is a means to fully suppress leaching also by using a non-TC resist.

Segregation is a very unstable physical phenomenon that must be controlled in order to use a non-TC resist. Although a number of analysis results have been reported about the segregation layer of a non-TC resist $[8,9]$, the characteristics of the segregation layer of a hydrophobic additive have still not been sufficiently understood.

In this study, some non-TC resists were made using a model base polymer, PAG, and hydrophobic additives. The characteristics of the segregation layer were thoroughly investigated using various analyses. Furthermore, it was investigated when the segregation would be generated. Finally, the state of an ideal segregation layer was considered.

\section{Experimental}

\subsection{Materials}

In order to check the state of segregation layer of a non-TC resist, a FUGU polymer and a FUGU-MOM polymer (shown in Fig. 1 (a) and (b), respectively) were used as hydrophobic additives. The FUGU polymer had partially fluorinated monocyclic structure and hexafluoroalcohol (HFA) [10]. The FUGU-MOM polymer was a FUGU polymer protected by a methoxymethyl protecting group.

Further, in order to determine the step at which the segregation phenomenon had occurred, a FUGU/FNBMA polymer which was copolymer of FUGU with methacrylate monomer FNBMA (Fig. 1 (c)) was used as a hydrophobic additive. The characteristics are suitable for practical use: it exhibits both developer solubility and hydrophobicity.

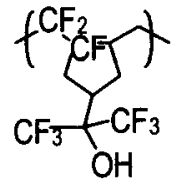

(a)

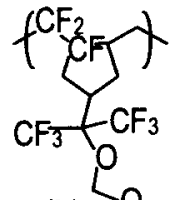

(b) 0

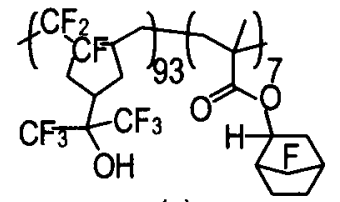

(c)
Fig. 1. Chemical formula of (a) FUGU additive,

(b) FUGU-MOM additive, and (c) FUGU/FNBMA additive.

All these additives were manufactured by Asahi Glass Co., Ltd. Table 1 shows the contact angle and the developer dissolution rate for the coating films of these additives.

Table 1. Contact angle and developer dissolution rate of additive coating film.

\begin{tabular}{cccc}
\hline Additive & $\begin{array}{c}\text { CA } \\
\left({ }^{\circ}\right)\end{array}$ & $\begin{array}{c}\text { RCA } \\
\left({ }^{\circ}\right)\end{array}$ & $\begin{array}{c}\text { Dissolution rate } \\
(\mathrm{nm} / \mathrm{s})\end{array}$ \\
\hline (a) & 70 & 60 & 400 \\
(b) & 90 & 89 & 0 \\
(c) & 97 & 79 & 100 \\
\hline Base polymer & 70 & 55 & 0 \\
\hline
\end{tabular}

The methacrylic resin shown in Fig. 2 was used as the base polymer of the non-TC resists; triphenylsulfonium perfluorobutanesulfonate (TPS-PFBS), as PAG; and propylene glycol monomethyl ether acetate (PGMEA), as the solvent. A non-TC resist was made by mixing the base polymer, a hydrophobic additive, PAG, and the solvent.

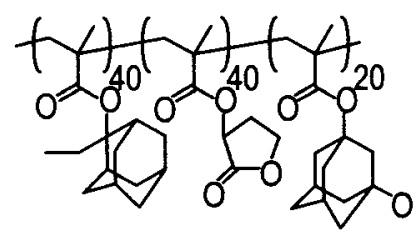

Fig. 2. Chemical formula of base polymer.

\subsection{Experimental conditions}

(a) Film stack

The segregation of a hydrophobic additive is influenced by not only the surface free energy of a resist but also the interfacial energy of the interface with the lower film. Therefore, it is preferred for the segregation evaluation to use the lower film actually used. A silicon-containing organic film that we used in the multilayer process was used as the lower film in this study. Its thickness was 72 $\mathrm{nm}$. The film thickness of the non-TC resist 
was set as $150 \mathrm{~nm}$, except for the evaluation part in which the rotation frequency of spin coating was intentionally changed.

(b) Contact angle measurement

The static and the dynamic contact angles of the non-TC resists were measured using two types of devices: (1) Model CA-X (Kyowa Interface Science Co., Ltd.), employing the extension/contraction method. (2) DM-700 (Kyowa Interface Science Co., Ltd.), employing the sliding angle method.

(c) TOF-SIMS analysis

In order to analyze the chemical composition of the segregation layer in a non-TC resist, time-of-flight secondary ion mass spectrometry (TOF-SIMS) analysis was performed. For depth profile analysis, the resist was diagonally cut by gradient shaving preparation (GSP) [11], and TOF-SIMS analysis was executed on the cross section. TOF-SIMS 5 (ION-TOF GmbH) was used for the TOF-SIMS analysis. $\mathrm{Bi}_{3}{ }^{2+}$ was used as the primary ion; its irradiation dose was adjusted to $5 \mathrm{E} 11 \mathrm{ions} / \mathrm{cm}^{2}$ or less and the acceleration voltage was $25 \mathrm{kV}$.

\section{(d) XPS analysis}

The elemental depth profile of a non-TC resist was measured using $\mathrm{X}$-ray photoelectron spectroscopy (XPS). A PHI Quantera SXM (ULVAC-PHI, Inc) with AlK $\alpha$ excitation was used for the XPS analysis. A 2 $\mathrm{mm} \times 2 \mathrm{~mm}$ area of the non-TC resist was sputtered using the $\mathrm{C}_{60}{ }^{+}$ions accelerated by a voltage of $10 \mathrm{kV}$.

(e) Film density measurement

The film density of a non-TC resist was determined using grazing incidence $\mathrm{x}$-ray reflectometry (GIXR). In this examination, the film density was evaluated from the total-reflection critical angle of the incident $\mathrm{X}$-rays. Since this angle depends on the refractive index of the top surface, the film density calculated here is, in fact, the film density of the top surface. For GIXR, RINT-2400 (Rigaku Corporation) was used. The wavelength of the incident $\mathrm{X}$-rays was $0.15406 \mathrm{~nm}$, and the angle of incidence was $0.05^{\circ}-2.0^{\circ}$.

The measurements (c)-(e) were performed by Toray Research Center Inc.

\section{Results and Discussion}

3.1. What is the state of the segregation layer of a non-topcoat resist?

The state of the segregation layer of resist with each the FUGU additive and the FUGU-MOM additive was investigated. First, TOF-SIMS analysis was performed for various depths. Fig. 3 shows the secondary-ion intensity of the hydrophobic additive of each resist.

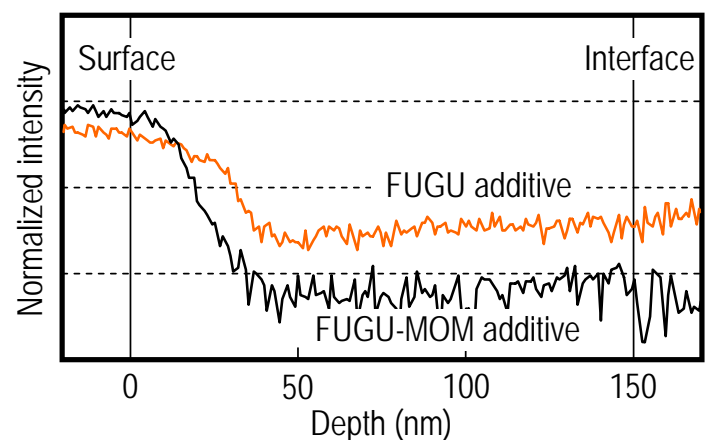

Fig. 3. Secondary-ion intensity of hydrophobic additive, determined using TOF-SIMS.

With regard to the resist with the FUGU additive, the secondary-ion intensity of the additive on the top surface was smaller and that in the bulk was larger than the corresponding values of the resist with the FUGU-MOM additive. It was speculated that the surface of the resist with the FUGU additive was not completely covered by the additive and that the additive remained in the bulk. Although this tendency qualitatively agrees well with the above TOF-SIMS result, the quantitative evaluation is difficult because the ionization efficiency strongly depends on the characteristics of the material.

Hence, next, the elemental depth profiles of these non-TC resists were measured using XPS. In order to reduce the resist film damage by ion etching, it was etched using a large ionic species, $\mathrm{C}_{60}{ }^{+}$, and XPS measurements were made (Fig. 4).

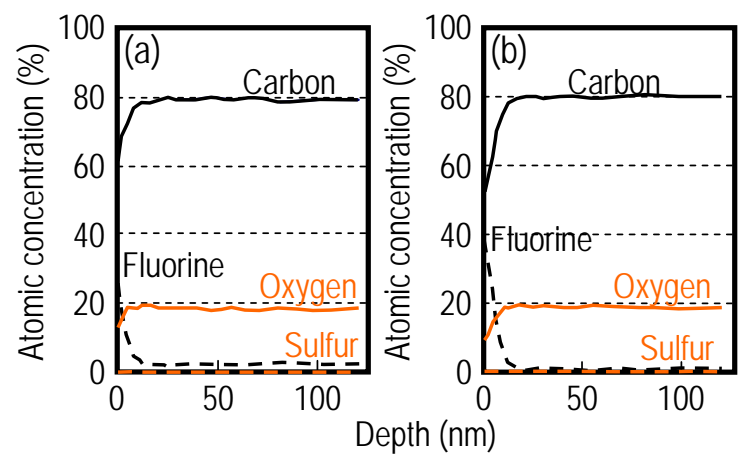

Fig. 4. Elemental depth profile of resist with (a) FUGU additive and (b) FUGU-MOM additive, determined using XPS.

The atomic ratios determined from the XPS results were converted into the mol ratios of the base polymer, hydrophobic additive, and PAG by fitting. Attention was paid to the base polymer and the additive with a large molecular weight, and the ratio is 
shown in Fig. 5.

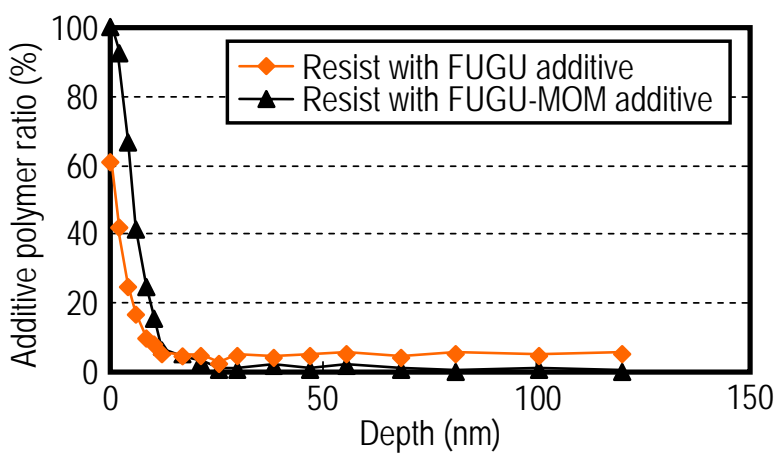

Fig. 5. Ratio of hydrophobic additive in resist with FUGU additive and FUGU-MOM additive.

From this result, the segregation layer of the resist with the FUGU additive was understood to be as follows:

- In the composition ratio of the top surface of the resist, the hydrophobic additive was $61 \%$, and base polymer was $39 \%$.

- The thickness of the segregation layer was about $17 \mathrm{~nm}$.

- About $4.6 \%$ of the hydrophobic additive remained in the bulk.

On the other hand, the segregation layer of the resist with the FUGU-MOM additive was understood to be as follows:

- In the composition ratio of the top surface of the resist, the hydrophobic additive was $100 \%$. The fluorine ratio of the top-surface of the resist was larger than that of the hydrophobic additive; this result contradicts the fact that among the materials used for preparing the resist, the additive contained most fluorine. Therefore, it may appear that the fluorine-containing part of the additive was preferentially oriented toward the surface.

- The thickness of the segregation layer was about $25 \mathrm{~nm}$.

- The hydrophobic additive hardly remained in the bulk.

These results indicate that a segregation layer comprising only a hydrophobic additive could be formed if the hydrophobic additive had a low critical surface tension. However, if the hydrophobic additive had a high critical surface tension, the segregation layer in which the base polymer and the additive were intermingled was formed and the additive remained in the bulk also.

Table 2 shows the leaching of PAG cations into the water and the receding contact angle (RCA) of each resist. The hydrophobicity of the segregation layer of each resist is almost equal to that of the coating film of the hydrophobic additive. However, in the resist with the FUGU additive, since the base polymer was exposed to the top-surface, leaching was not sufficiently suppressed. On the other hand, in the resist with the FUGU-MOM additive since the top surface was covered by the additive, leaching of PAG was hardly observed.

Table 2. Receding contact angle and PAG cation leaching of non-TC resist.

\begin{tabular}{ccc}
\hline Coating film & $\begin{array}{c}\text { RCA } \\
\left({ }^{\circ}\right)\end{array}$ & $\begin{array}{c}\text { PAG cation leaching } \\
\left(\mathrm{mol}^{2} / \mathrm{cm}^{2} / \mathrm{s}\right)\end{array}$ \\
\hline $\begin{array}{c}\text { Resist with } \\
\text { FUGU additive }\end{array}$ & 60 & $2.21 \mathrm{E}-13$ \\
\hline $\begin{array}{c}\text { Resist with } \\
\text { FUGU-MOM additive }\end{array}$ & 88 & $2.67 \mathrm{E}-16$ \\
\hline Base polymer & 55 & $2.46 \mathrm{E}-13$ \\
\hline
\end{tabular}

The following two reasons could be considered to explain why the segregation layer was different depending on the type of the additive used. One reason is that the critical surface tension of an additive itself changes according to its polar groups. The segregation layer comprising only a hydrophobic additive could be easily formed in the case that the critical surface tension of the hydrophobic additive was low and the difference of the critical surface tension between the base polymer and the hydrophobic additive was large. Another reason is the influence of the compatibility between the base polymer and the additive: it is difficult to separate them if the compatibility is high. Since both the FUGU polymer and the base polymer had a hydroxyl group in the end, they were speculated to have high compatibility.

The top surface of the resist with the FUGU-MOM additive was the segregation layer that comprised only the FUGU-MOM additive. Now, we address the following question: Were the characteristics of the top surface of the segregation layer similar to those of the coating film? The densities of the coating film and the top surface of the segregation layer were measured using GIXR (Fig. 6). In general, the film density of the coating film with the fluorine compound was higher than that of the coating film of the conventional resist base-polymer. The coating film of the FUGU-MOM polymer had a high film density. 


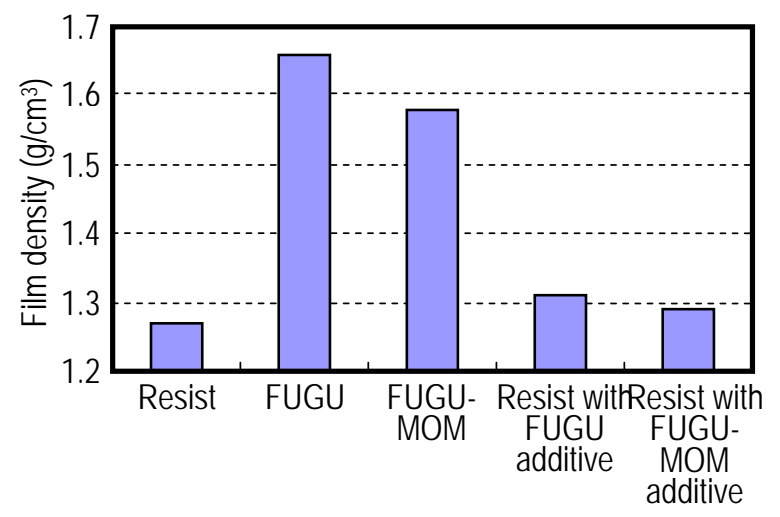

Fig. 6. Top-surface film density of resist, additive coating film, and non-TC resist measured by GIXR.

The top surface of the resist with FUGU-MOM additive exhibited low film density comparable to the resist base-polymer, although it was the segregation layer that comprised only the FUGU-MOM polymer. The film density of the segregation layer might become low by its interaction with the base polymer. Since the film density of the segregation layer was lower than that of the coating film, the segregation layer might not suppress leaching as effectively as that suppressed by an equally thick coating film. If a base polymer of a high film density can be used, the film density of the segregation layer may also become high and its performance may improve further.

3.2. When did the segregation of a hydrophobic additive in a non-TC resist occur?

In this section, it is verified in which process step the segregation of a hydrophobic additive occurred. FUGU/FNBMA polymer (Fig. 1 (c)) was used as the hydrophobic additive. First, it was expected that the segregation was induced by thermal energy. The pre-bake temperature of the non-TC resist was changed, and each contact angle was compared. After the resist was spin coated at a rotation frequency of $500 \mathrm{rpm}$, the contact angles of unbaked and baked $\left(110^{\circ} \mathrm{C}\right.$ for 60s) samples were measured (Fig. 7 (a)). If segregation occurs during baking, the contact angle should increase after baking. However, no difference was observed in the contact angles of the baked and unbaked samples. In addition, no difference was observed in their secondary ion intensities, measured by TOF-SIMS analysis (Fig. 8). Furthermore, no correlation was seen between the baking conditions and the contact angle, although change in baking temperature and dual baking were employed (Fig. 7 (b)).

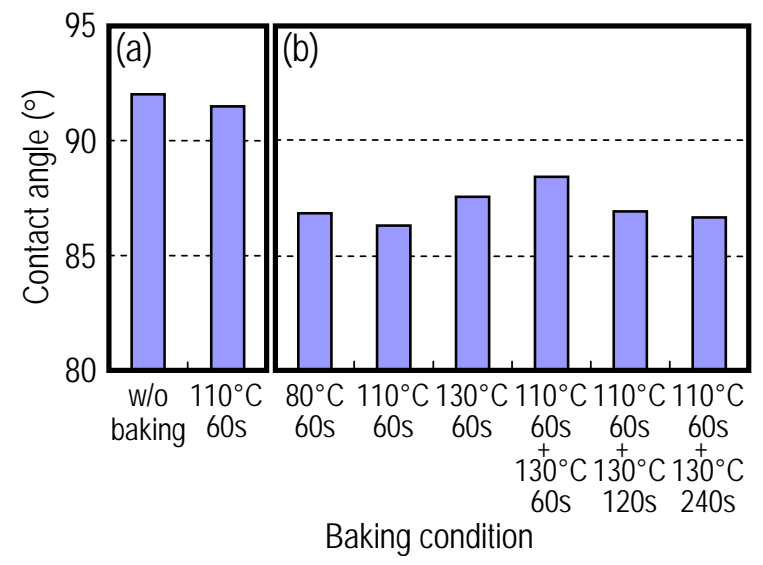

Fig. 7. Baking-condition dependency of contact angle of resist with FUGU/FNBMA additive coated at (a) $500 \mathrm{rpm}$ and (b) $4000 \mathrm{rpm}$.

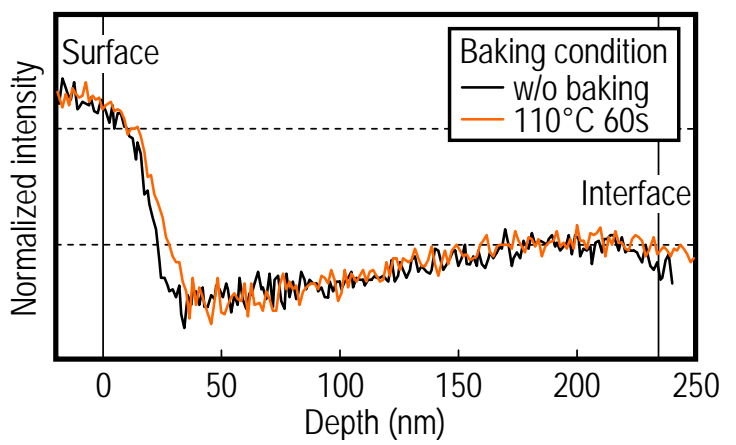

Fig. 8. Baking-condition dependency of additive secondary ion intensity by TOF-SIMS of resist with FUGU/FNBMA additive (rotation frequency: $500 \mathrm{rpm})$.

These results showed that the segregation was not induced by the thermal energy of pre-baking. Fig. 9 shows that the static contact angle of the non-TC resist decreased with an increase in the rotation frequency of spin coating.

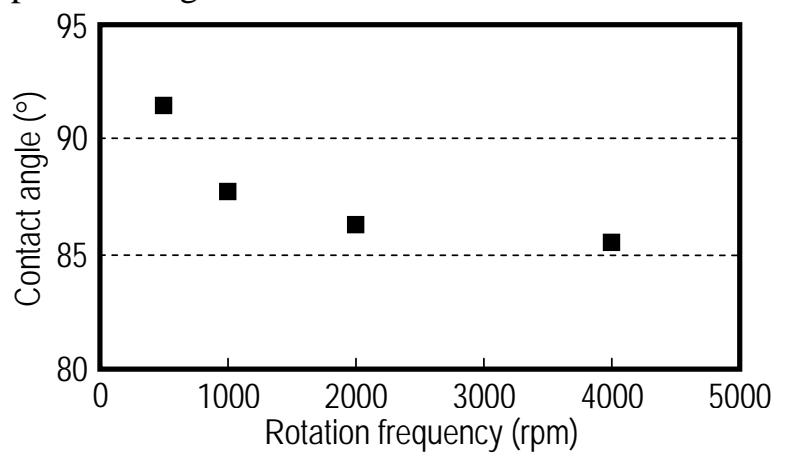

Fig. 9. Coating rotation frequency dependency of contact angle of resist with FUGU/FNBMA additive (baking condition: $110^{\circ} \mathrm{C} 60 \mathrm{~s}$ ) .

Hence, the segregation of the hydrophobic additive almost entirely occurs during spin 
coating. A change in the rotation frequency influenced the centrifugal force and the film thickness. It was investigated whether a change in the centrifugal force or that in the film thickness influenced the contact angle.

First, the difference in the additive segregation of the wafer central part and outer part was evaluated. The centrifugal force at the outer part is considerably larger than at the central part of the wafer. If a change in the centrifugal force influenced the segregation of the hydrophobic additive, there must be a difference in the segregation in the wafer. However, there was little difference in the additive segregation between the central and outer parts of the wafer (Fig. 10).

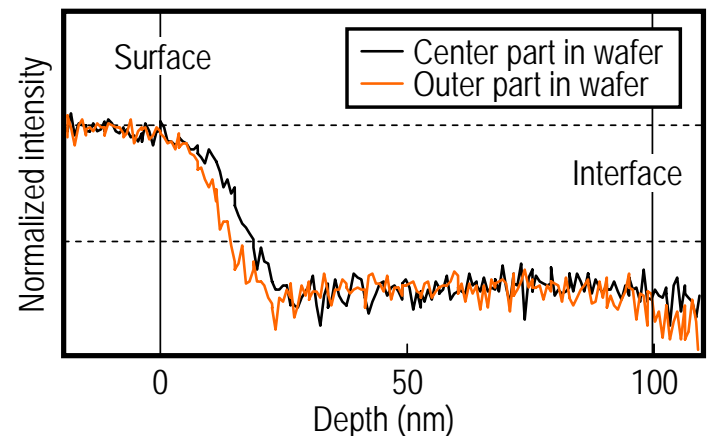

Fig. 10. Secondary-ion intensity comparison by TOF-SIMS of the FUGU/FNBMA additive of resist between central and outer parts of wafer (rotation frequency: $500 \mathrm{rpm}$, baking condition: $\left.110{ }^{\circ} \mathrm{C} 60 \mathrm{~s}\right)$.

Next, the resist solution was further diluted with the solvent, and a few films were prepared. The contact angles of the films prepared using the undiluted and diluted resist solutions were compared for the same film thickness but different rotation frequencies (Fig. 11).

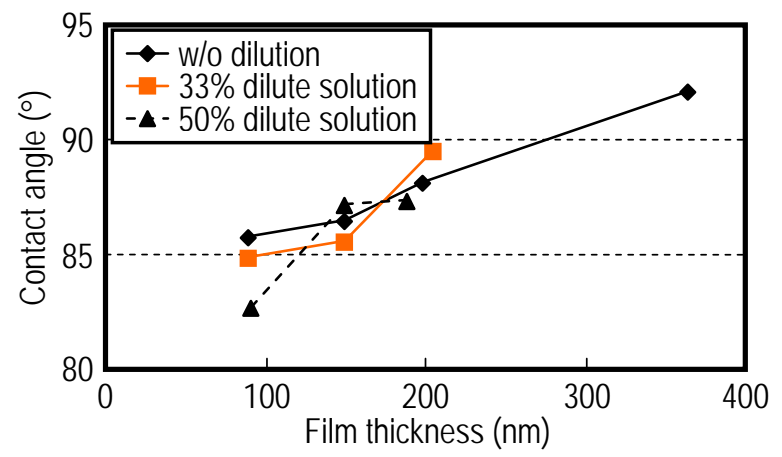

Fig. 11. Film-thickness dependency of contact angle of resist with FUGU/FNBMA additive (baking condition: $\left.110^{\circ} \mathrm{C} 60 \mathrm{~s}\right)$.
If the film thickness was the same regardless of the rotation frequency, the contact angle was nearly the same.

From the results mentioned in the above two paragraphs, it was considered that the contact angle decreases with an increase in the coating rotation frequency since the resist volume on the wafer decreases and the amount of the hydrophobic additive proportionately decreases. Many of the intermixed base polymer and hydrophobic additives are exhausted without segregation in the first stage of spin coating, and the surplus additive contained in the resist, which remained on the wafer, became segregated. Figure 12 shows the secondary-ion intensities of the hydrophobic additive, determined from the TOF-SIMS analysis of two resists comprising the FUGU/FNBMA additive: one resist was spin coated at a rotation frequency of $500 \mathrm{rpm}$; the other, at $4000 \mathrm{rpm}$.

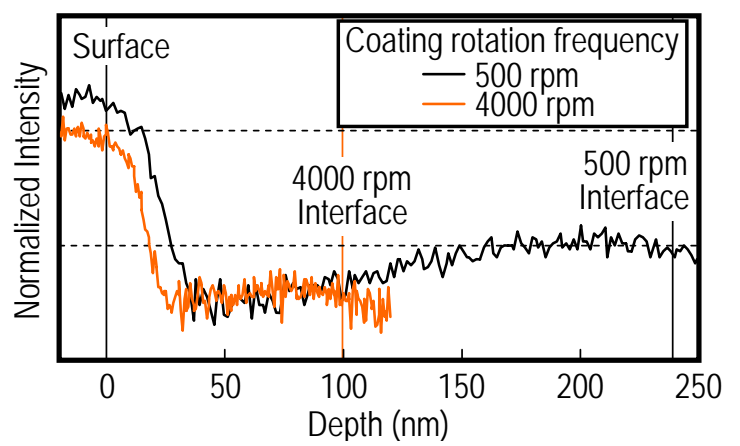

Fig. 12. Coating rotation frequency of additive secondary ion intensity by TOF-SIMS of resist with FUGU/FNBMA additive (baking condition: $110^{\circ} \mathrm{C}$ $60 \mathrm{~s})$.

Because the resists were prepared from the same solution but at different rotations frequencies, they had different thicknesses. The secondary-ion intensity of the additive on the top surface of the sample spin-coated at $4000 \mathrm{rpm}$ was lower than that spin coated at 500 rpm. The TOF-SIMS results also confirmed that the amount of the hydrophobic additive on the top surface decreased with an increase in the rotation frequency. In the case of the sample spin coated at $4000 \mathrm{rpm}$, the additive formed a segregation layer that extended from the surface up to a depth of 25 $\mathrm{nm}$. And the concentration of the additive became almost constant at a depth of $25 \mathrm{~nm}$ or more. On the other hand, in the case of the sample spin coated at $500 \mathrm{rpm}$, the additive formed a segregation layer that extended from the surface up to a depth of $45 \mathrm{~nm}$. In the depth region from $45 \mathrm{~nm}$ to $175 \mathrm{~nm}$ of the 
resist, the concentration of the additive increased with the depth and became almost constant at a depth of $175 \mathrm{~nm}$ or more.

According to the mean field theory, the volume fraction of a hydrophobic additive, $\phi(z)$, at a depth, $z$, is approximated by the following equation $[12,13]$.

$$
\phi(z)=\phi b+(\phi s-\phi b) \exp (-z / \zeta)
$$

where $\phi b$ and $\phi s$ are the volume fractions of the hydrophobic additive in the bulk and the surface, respectively, and $\zeta$ is the surface decay length. According to equation (1), the volume fraction exponentially decreases from the surface to the bulk and becomes constant in the bulk. Although the secondary-ion intensities of the additive had a local minimum point in Fig. 12, the volume fractions of the additive did not have a local minimum point in equation (1). The following reason could be considered to explain why a local minimum point existed in Fig. 12.

Because the segregation phenomenon that minimized the surface free energy was fast, the segregation was considered to occur in the first stage of spin coating. When the segregation occurred, a concentration gradient of the additive was generated between the bulk and the depth at which the additive concentration was lowered by the segregation. Next, the additive diffused in order to lower the concentration gradient. In the case that the solvent evaporated and it became impossible to move the additive before the diffusion of the additive reached the equilibrium, it was speculated that the minimum concentration of the additive appeared in the film. It was thought that the minimum concentration of the additive did not exist in the film, as shown by equation (1) if the overall resist was in equilibrium by diffusion.

Since the mobility of the additive in a film naturally changes with the amounts of the residual solvents, the concentration distribution of the additive in the film was expected to change with the coating conditions. Moreover, it was expected that the concentration distribution changed with the solvent characteristics such as the viscosity and volatility. If the segregation phenomenon was sufficiently fast, the coating conditions might not influence the surface characteristic of the non-TC resist so greatly. However, in the case that the additive remained in the bulk and its concentration distribution in the film changed with the coating conditions, there was a possibility that the pattern profile or the resist characteristics might be greatly affected by the coating conditions. After all, it was not desirable that the hydrophobic additive remained in the resist bulk for the non-TC resist.

From the above results, it was considered that the film thickness of a non-TC resist should be such that the amount of the hydrophobic additive was sufficient to form the segregation layer and the additive did not remain in the bulk. We thought that the segregation of the additive was determined by the critical surface tension difference and the compatibility between the additive and the base polymer. That is, a suitable film thickness for practical use differs with the kind of the hydrophobic additive and the base polymer.

In this result, the segregation mostly occurred during spin coating. However, in some materials, the segregation speed may be slow. In such a case, it is expected to change the state of the segregation layer, depending on the coating condition. In order to form a stable segregation layer, the selected materials and coating conditions should be such that they lead to the complete segregation during spin coating

\subsection{Desirable hydrophobic additive and its} usage

The characteristics required for a hydrophobic additive and its usage are summarized as follows.

- It is desirable that a segregation layer comprising only the hydrophobic additive form.

- The additive should have a low critical surface tension.

- It should have good solubility in a developer so that it does not form developer insoluble defects. It is desirable that it in unexposed area . also dissolves in the developer.

- In order to minimize the risk of forming developer insoluble defects, the amount of the additive should be as less as possible. Very high developer solubility cannot be expected of the additive of a low critical surface tension.

- In order to achieve better segregation using a small amount of additive, it should have low compatibility with the base polymer.

- In order to obtain a stable segregation layer, it is desirable to select a material that has a high segregation speed as the segregation is complete during spin coating. The condition 
that a residual solvent exists in the resist film might be effective in the stabilization of the segregation layer.

- According to the structures and the amounts of the base polymer and the additive, the resist film thickness must be optimized. If the film is very thin, the absolute amount of the additive becomes insufficient. On the other hand, if the film is very thick, the additive remains in the bulk and affects the resist profile and characteristics.

And although it has not yet been confirmed, if a base polymer with a high film density is used, the film density of the segregation layer may also become high due to its interaction with the base polymer. We believe that an increase in the film density of the segregation layer contributes to suppress leaching and improve hydrophobicity.

\section{Summary}

In this study, the characteristics of the segregation layer of a non-TC resist were investigated. The results indicated that a segregation layer comprising only the hydrophobic additive could form if the additive had low critical surface tension. However, the segregation layer differed from the coating film and exhibited a film density comparable to that of the base polymer. Moreover, it was understood that the segregation of the additive mostly occurred during spin coating. The film thickness of a non-TC resist must have the optimum: a thin film exhibited low hydrophobicity, whereas the additive remained in the resist bulk for a thick film. Furthermore, there were no differences in the segregation layer in the central and outer parts of the wafer.

From these results, the ideal form of a non-topcoat resist is quite clear. We should surely avoid damaging very expensive immersion scanner. When using a non-TC resist, it is necessary to understand the characteristics and note the usage. If it is used with sufficient understanding, it is sure that a non-TC resist can reduce the cost and increase the throughput safely.

\section{References}

1. W. Taberelli and E. Loebach, U.S. Patent No. 4,346,164, (1982).

2. S. Mimotogi, F. Uesawa, M. Tominaga, $H$. Fujise, K. Sho, M. Katsumata, H. Hane, A. Ikegami, S. Nagahara, T. Ema, M. Asano, H. Kanai, T. Kimura and M. Iwai, Proc.SPIE, 6520 (2007), 652008-1.

3. K. Ohmori, T. Ando, T. Takayama, K. Ishizuka, M. Yoshida, Y. Utsumi, K. Endo and T. Iwai, Proc. SPIE, 6153 (2006), 61531X-1.

4. R. D. Allen, P. J. Brock, L. Sundberg, C. E. Larson, G. M. Wallraff, W. D. Hinsberg, J. Meute, T. Shimokawa, T. Chiba and M. Slezak, J. Photopolym. Sci. \& Tech., 18 (2005), 615.

5. T. Ishibashi, T. Hanawa, T. Suganaga, S. Maejima, K. Narimatsu, K. Suko, M. Terai, T. Kumada and T. Ando, J. Photopolym. Sci. \& Tech., 19 (2006), 547.

6. N. Stepanenko, H. yun-Woo Kim, S. Kishimura, D. Van Den Heuvel, N. Vandenbroeck, M. Kocsis, P. Foubert, M. Maenhoudt, M. Ercken, F. Van Roey, R. Gronheid, I. Pollentier, D. Vangoidsenhoven, C. Delvaux, C. Baerts, S. O'Brien, W. Fyen, G. Wells, Proc. SPIE, 6153 (2006), 615304-1.

7. N. Shirota, Y. Takebe, S. Wang, T. Sasaki, O. Yokokoji, Proc. SPIE, 6519 (2007), 651905-1.

8. I. Takemoto, N. Ando, K. Edamatsu, Y. Fuji, K. Hashimoto, J. Funase and $\mathrm{H}$. Yokoyama, J. Photopolym. Sci \& Tech., 20 (2007), 473.

9. K. Wada, S. Kanna and H. Kanda, Proc. SPIE, 6519 (2007), 651908-1.

10.Y. Takebe, M. Eda, S. Okada, O. Yokokoji, S. Irie, A. Otoguro, K. Fujii and T. Itani, Proc. SPIE, 5376 (2004), 151.

11. N. Man, H. Okumura, H. Oizumi, N. Nagai, H. Seki, I. Nishiyama, Applied surface science, 231-232 (2004), 353.

12. C. Forrey, J. T. Koberstein, D. H. Pan, Interface Science, 11 (2003), 211.

13. K. Tanaka, D. Kawaguchi, A. Takahara, T. Kajiyama, Function \& Materials, 23 (2003), 19. 